\title{
Normocomplementemic Urticarial Vasculitis Associated with A/H1N1 in a Child. Case Report
}

\author{
Şule Gökçe ${ }^{1}$ (D) • Burçe Emine Dörtkardeşler ${ }^{1}$ • Aslı Aslan ${ }^{2}$
}

Accepted: 19 November 2020 / Published online: 25 November 2020

(C) Springer Nature Switzerland AG 2020

\begin{abstract}
Urticarial vasculitis is an entity characterized by urticarial rashes that are typically pruritic and painful, associated with angioedema and purpura. Influenza viruses are common respiratory pathogens that can cause seasonal infections and global human morbidity/mortality. A 4.5-year-old girl presented with fever and painful urticarial skin lesions leaving post-inflammatory hyperpigmentation. The nasal specimens showed a positive for influenza A/H1N1. On the basis of these findings, a diagnosis of urticarial vasculitis associated with $\mathrm{A} / \mathrm{H} 1 \mathrm{~N} 1$ was concluded. Taking the results together, we suggest that urticarial vasculitic lesions can be considered to the list of cutaneous manifestations during the seasonal flu.
\end{abstract}

Keywords Child $\cdot$ Vasculitis $\cdot$ Urticarial vasculitis $\cdot$ Influenza infection

\section{Introduction}

Generally, influenza infection is an acute, self-limited, and uncomplicated disease that is caused by influenza A and B, and occurs every winter season [1]. Abrupt onset of fever, headache, myalgia, cough, sore throat, and rhinitis is mentioned as frequent clinical manifestations of uncomplicated influenza infections $[1,2]$. Cutaneous involvement with both influenza infection and vaccination has been reported as a macular/maculopapular rash or vasculitic/purpuric eruption [3]. Urticarial vasculitis (UV) is a type of leukocytoclastic vasculitis that may present with a generalized urticarialappearing eruption, fever, and arthralgias. This is a

This article is part of the Topical Collection on Medicine

Șule Gökçe

sule.gokce@ege.edu.tr

Burçe Emine Dörtkardeșler

burce.dortkardesler@ege.edu.tr

Asli Aslan

drasliaslan@gmail.com

1 Ege University Faculty of Medicine, Department of Pediatrics, General Pediatrics Unit, Ege University Childrens' Hospital, Bornova, 35040 Izmir, Turkey

2 Department of Medical Genetics, Faculty of Medicine, Ege University, 35100 Izmir, Turkey clinicopathologic entity thought to be mediated by a TypeIII hypersensitivity reaction mediated by antigen-antibody complexes deposited on vascular endothelium creating inflammation and vasculitis. Urticarial lesions are typically fixed for more than $24 \mathrm{~h}$ and may be associated with petechiae, purpura, or post-inflammatory hyperpigmentation. The prevalence is unknown and occurs in middle-aged women being rare in children $[3,4]$. Leukocytoclastic vasculitis following seasonal influenza vaccination has been reported in several cases [5]. However, urticarial vasculitis associated with $\mathrm{A} / \mathrm{H} 1 \mathrm{~N} 1$ infection has not been reported previously. This case describes a 4.5-year-old girl who presented with fever and painful urticarial skin lesions, and diagnosed with A/H1N1 associated with urticarial vasculitis.

\section{Case Report}

A previously healthy 4.5 -year-old girl was admitted to our outpatient department with a 6-day history of nonproductive cough, nasal congestion, myalgias, and fever with a temperature of $39{ }^{\circ} \mathrm{C}$ twice a day during the period of seasonal flu. After a 3-day history of flu symptoms, urticarial lesions were noticed on various parts of the body mostly the face and upper extremities, accompanied by arthralgia, edema, and pain on hands and wrist. It was found out that the patient was diagnosed with "urticaria" and was treated with antihistaminic treatment in another hospital 3 days before. In the last 2 days, 
the urticarial rash turned a purpuric morphology with central clearing. Urticarial plaques persisted over $24 \mathrm{~h}$ with pain and burning sensation, and resolved with hyperpigmentation. The child was fully immunized according to the Turkish immunization program. There was no family history or past history of atopic diseases, or history of using any medication and vaccine implementation. On admission, her physical examination parameters were as follows: temperature $38.2{ }^{\circ} \mathrm{C}$, the pulse 110 beats per minute, and blood pressure $96 / 61 \mathrm{mmHg}$. She was conscious but suffered from itching in various parts of the body and pain and edema/tenderness on the hands, wrist of hands, and ankles. The pain increased when the edematous areas are touched. On examination, there was an effervescent urticarial rash with associated bilaterally hand edema which on resolution left a violaceous-brownish purpuric lesion. Some urticarial rashes subsided, leaving post-inflammatory hyperpigmentation. The following laboratory evaluations were within the normal ranges: hemoglobin, white cells, platelets, electrolytes, renal function, international normalized ratio, activated partial thromboplastin count, erythrocyte sedimentation rate, C-reactive protein, immunoglobulin levels, complement (C3, C4, C1q), and rheumatoid factor. There was no blood or protein in the urine. Subsequent antibody tests were negative. The serology screening for infections revealed that the Epstein-Barr virus (EBV), hepatitis A-B-C, human immunodeficiency virus (HIV), cytomegalovirus (CMV), and parvovirus B19 serology were negative. Specific laboratory tests were performed ruling out immunological and autoimmune disorders, including anti-nuclear antibody profile, anti-smooth muscle, anti-liver/kidney microsomal antibodies, anti-cardiolipin IgG and IgM, antineutrophilic cytoplasmic antibodies (ANCA), lupus anticoagulant, and rheumatoid factor. Chest radiographs were taken with no pathological findings. Blood and urine cultures were bacteriologically sterile. Polymerase chain reaction (PCR) assays of nasopharyngeal swab specimen for adenovirus, enterovirus, rhinovirus, respiratory syncytial virus, parainfluenza virus, human bocavirus, human metapneumovirus, and coronavirus were all negative. However, A/H1N1 was detected in a nasopharyngeal swab specimen by multiplex PCR. The patient was diagnosed with A/H1N1-associated urticarial vasculitis based on the patient's history and clinical findings. The patient was given oseltamivir $17 \mathrm{mg}$ orally twice daily for 5 days, dual antihistaminic drugs, and low doses of systemic corticosteroids for 5 days. Histopathological examination showed a marked presence of vascular endothelium and a slight level of perivascular lymphocyte infiltration with edema in the papillary dermis. All of the patient's remaining symptoms resolved over the next 4 days. Although the cutaneous urticarial lesions and fever showed excellent regression, the violaceous-brownish purpuric lesions regressed leaving hyperpigmentation for 7 days. The patient was prescribed anti-histamines for 15 days.
After 2 weeks, the entity was totally resolved without scarring or residual hyperpigmentation. In the following 2 months, no new active skin lesions were detected.

\section{Discussion}

This is an unusual presentation of H1N1 influenza A infection presenting with findings of urticarial vasculitis. The clinical reports published to date have not demonstrated this pattern of cutaneous manifestation; however, it was likely secondary to an influenza vaccine. Dermatological manifestations due to influenza infections and influenza vaccines include a variety of conditions such as a rash (macular-maculopapular), purpura, and petechiae. There have been reported that petechial eruptions were seen in 3-10\% of children infected with pandemic H1N1 influenza [6]. However, vasculitic-purpuric syndromes are an extremely rare manifestation in influenza infection. Silva ME et al. [5] described a case of a child with a febrile petechial rash associated with influenza A virus infection. Rocco Urso et al. [6] have reported a 23-year-old Caucasian woman was hospitalized for fever, severe abdominal pain, hematuria, arthritis, and purpuric manifestations associated with pandemic 2009 (H1N1) virus by reversetranscriptase PCR assay. A report conducted in the USA showed a case with vaso-occlusive retinal vasculitis following H1N1 infections [7]. Regarding the systematic review of current literature for influenza-associated vasculitis in children, we searched for articles published up to 2010 in the following databases: PubMed, Medline, Embase, Cochrane Library, and CINAHL. We used search terms including "influenza," "childhood," "vasculitis," and "urticarial vasculitis" to identify reports that presented data on influenza-associated vasculitis. To our knowledge, there are no reports concerning cases of urticarial vasculitis associated with the A/H1N1 infection.

Urticarial vasculitis, first described by McDuffie in 1973, is a clinicopathologic entity characterized by urticarial rashes that are typically pruritic and painful, associated with angioedema and purpura [8]. The urticarial plaques of UV persist for more than $24 \mathrm{~h}$, can have a central dark red or brown macule, and may resolve with residual purpura or hyperpigmentation. Typical UV criteria are urticarial lesions lasting more than $24 \mathrm{~h}$ and 2 of 3 of the following: pain or tenderness, purpura, or resolution with hyperpigmentation. The histopathological spectrum of urticarial vasculitis shows from minimal vascular damage to severe leukocytoclastic vasculitis caused by endothelial cell damage, fibrin deposition, and acellular infiltrate of neutrophils with or without leukocytoclastic and lymphocytes. The pathophysiology of UV is thought to be a formation of immune complexes in the blood that then deposits in vessel walls due to third-type hypersensitivity reaction against any of antigens [9]. 
For patients evaluated because of the novel 2009 H1N1 influenza infection, the incidence of rash is about $2 \%$ typically macular or maculopapular in children [10]. Ryan-Poirier reported that macular, maculopapular, and petechial rashes appear in a few counts of children with influenza virus infections. The classic presentations of influenza A virus infection consist the sudden onset of fever, chills, myalgia, cough, sore throat, rhinorrhea, croup, bronchitis, bronchiolitis, and pneumonitis [11]. Despite the documentation of UV following influenza vaccination, no report has described an association of UV with influenza infection.

In conclusion, we have reported the first case of UV associated with influenza A virus infection diagnosed by clinical features and proven by skin biopsy, treated with oseltamivir, antihistaminic drugs, and prednisolone. Influenza virus can also sometimes be associated with dermatologic findings, and therefore, such associations should be kept in mind during seasonal influenza infection.

Authors' Contributions Ș.G.: contributed to acquisition, analysis, or interpretation of data. S..G., B.E.D., A.A..: critically revised the manuscript for important intellectual content. A.A.: agreed to be accountable for all aspects of the work in ensuring that questions relating to the accuracy or integrity of any part of the work are appropriately investigated and resolved. The manuscript has been read and approved by all authors.

\section{Compliance with Ethical Standards}

Conflicts of Interest The authors declare that they have no conflict of interest

Human and Animal Rights and Informed Consent This article does not contain any studies with human or animal subjects performed by any of the authors. Informed consent was taken from parents.

\section{Consent to Participate Informed consent was taken from parents.}

Consent for Publication The manuscript has not been submitted or accepted elsewhere. If accepted, the paper will not be published elsewhere in the same form, in English or in any other language, without written consent of the copyright holder.

\section{References}

1. Harper SA, Bradley JS, Englund JA, File TM, Gravenstein S, Hayden FG, et al. Seasonal influenza in adults and children-diagnosis, treatment, chemoprophylaxis, and institutional outbreak management: clinical practice guidelines of the infectious diseases society of America. Clin Infect Dis. 2009;8:1003-32. https://doi. org/10.1086/598513.

2. Silvennoinen H, Peltola V, Lehtinen P, Raija Vainionpää R, Heikkinen T. Clinical presentation of influenza in unselected children treated as outpatients. Pediatr Infect Dis J. 2009;5:372-5. https://doi.org/10.1097/INF.0b013e31819eef7.

3. Tavadia S, Drummond A, Evans CD, Wainwright NJ. Leucocytoclastic vasculitis and influenza vaccination. Clin Exp Dermatol. 2003;28:154-6. https://doi.org/10.1046/j.1365-2230. 2003.01188.x.

4. Moreno-Suarez F, Pulpillo-Ruiz A, Zulueta Dorado T, Conejo-Mir Sanchez J. Urticaria vasculitis: estudio retrospectivo de 15 casos. Actas Dermosifiliogr. 2013;104:579-85. https://doi.org/10.1016/j. adengl.2012.12.005.

5. Silva ME, Cherry JD, Wilton RJ, Bruckner DA, Miller MJ. Acute fever and petechial rash associated with influenza A virus infection. Clin Infect Dis. 1999;29:453-4. https://doi.org/10.1086/520240.

6. Urso R, Bevilacqua N, Gentile M, Biagioli D, Lauria FN. Pandemic 2009 H1N1 virus infection associated with purpuric skin lesions: a case report. J Med Case Rep. 2011;5:132. https://doi.org/10.1186/ 1752-1947-5-132.

7. Albert Y, Cheung BA, Stec L, Khandhar P, Williams GA. Bilateral vaso-occlusive retinal vasculitis with H1N1 influenza A infection. Retinal Cases Brief Rep. 2015;9:138-41. https://doi.org/10.1097/ ICB.0000000000118.

8. McDuffie FC, Jr Sams WM, Maldonado JE, Andreini PH, Conn DL, Samayoa EA. Hypocomplementaemia with cutaneous vasculitis and arthritis. Possible immune complex syndrome. Mayo Clin Proc. 1973;48:340-8.

9. Jachiet M, Flageul B, Deroux A, Le Quellec A, Maurier F, Cordoliani F, et al. The clinical spectrum and therapeutic management of hypocomplementemic urticarial vasculitis: data from a French nationwide study of fifty-seven patients. Arthritis Rheum. 2015;67:527-34. https://doi.org/10.1002/art.38956.

10. Lee JSS, Loh TH, Seow SC, Tan SH. Prolonged urticaria with purpura: the spectrum of clinical and histopathologic features in a prospective series of 22 patients exhibiting the clinical features of urticarial vasculitis. Am Acad Dermatol. 2007;56:994-1005. https://doi.org/10.1016/j.jaad.2006.10.962.

11. Ryan-Poirier K. Influenza virus infection in children. Adv Pediatr Infect Dis. 1995; 10:125-56.

Publisher's Note Springer Nature remains neutral with regard to jurisdictional claims in published maps and institutional affiliations. 